\title{
ALMOST LINEAR OPERATORS AND FUNCTIONALS ON $\mathcal{C}([0,1])$
}

\author{
J. R. BAXTER AND R. V. CHACON 1
}

ABSTRACT. Let $\mathcal{C}(M)$ be the bounded continuous functions on a topological space $M$. "Almost linear" operators (and functionals) on $\mathcal{C}(M)$ are defined. Almost linearity does not imply linearity in general. However, it is shown that if $M=[0,1]$ then any almost linear operator (or functional) must be linear. Specifically, if (a) $\|f\| \rightarrow 0$ implies $T(f) \rightarrow 0$, (b) $T(f+g)=T(f)+T(g)$ whenever $f g=0$, (c) $T(f+g)=T(f)+T(g)$ whenever $g$ is constant, and $M=[0,1]$, then $T$ is linear. An application is given to convergence of measures.

1. Introduction. A real-valued continuous function on the unit square can be thought of as a surface in three-dimensional space. Each continuous function is mapped to another continuous function by the following operation: Pour water over the surface until any hollows are filled and water begins to run off. Turn the resulting surface over and repeat the pouring, and then turn the new surface back to its original position. We will show in another paper how to put this description into mathematical terms. The resulting operator $T$ on continuous functions satisfies the following conditions:

(i) $\|T\| \leq 1$,

(ii) $T(f+g)=T(f)+T(g)$ provided that $f g=0$,

(iii) $T(\alpha f+\beta)=\alpha T(f)+\beta$, for $\alpha, \beta$ real numbers. $T$ is also monotone and continuous. Nonetheless $T$ is not linear.

For any topological space $M$, we call operators (or functionals) on $\mathcal{C}(M)$ which satisfy (i), (ii) and (iii) almost linear. It is our principal aim in this paper to show that almost linear operators (or functionals) on $\mathcal{C}(M)$ must in fact be linear if $M=[0,1]$. The operator result follows from the functional result by composing the operator $T$ with evaluation at a fixed but arbitrary point.

We in fact prove a little more since we show that (iii) may be replaced by the weaker condition

Received by the editors July 17, 1973 and, in revised form, November 20, 1973. AMS (MOS) subject classifications (1970). Primary 28A10; Secondary 28A25. ${ }^{1}$ Research supported by the National Science Foundation Grant GP 28683. 
(iii) $T(f+\beta)=T(f)+\beta$.

In $\$ 4$ we give an application of this stronger formulation to convergence of measures.

We also weaken (i) to (i)' $\lim _{\|f\| \rightarrow 0} \Phi(f)=0$.

Several authors have considered properties and representations of functionals and operators which have some of the characteristics of linearity but which may not in fact be linear. For example in [1]-[5] classes of functionals $T$ are considered which are such that if we define, for each $h \in C(M) \quad$ functional $T_{h}$ by setting $T_{h}(f)=T(f+b)-T(b), T_{h}$ satisfies (ii). In particular, $T(f)=\int f^{2}$ is such a functional. This functional does not, of course, satisfy (iii). We reserve the term almost linear for those operators or functionals which satisfy (i), (ii), and (iii), and it is those operators and functionals which are shown to be linear if $M=[0,1]$. The method of proof we use of course breaks down if the space has dimension greater than one. Lemmas 1 and 2 can be extended but Lemma 3 and its consequences fail.

2. Preliminaries. Let $\mathbf{R}$ be the real numbers. For any topological space $M$, let $\mathcal{C}(M)$ be the set of bounded continuous functions $f: M \rightarrow \mathbf{R}$. Let $\mathcal{C}_{0}(M)$ be the set of functions in $\mathcal{C}(M)$ with compact support. For any $f$ in $\mathcal{C}(M)$, let $\|f\|=\sup |f(x)|$, taken over all $x$ in $M$. We wish to prove:

Theorem 1. Let $\Phi: \mathcal{C}([0,1]) \rightarrow \mathbf{R}$ be a functional such that:

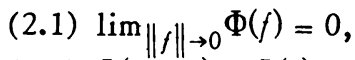

(2.2) $\Phi(f+g)=\Phi(f)+\Phi(g)$ whenever $f g=0$, and

(2.3) $\Phi(f+g)=\Phi(f)+\Phi(g)$ whenever $g$ is a constant. Then $\Phi$ is linear.

It is easy to see that (2.1) and (2.3) imply that $\Phi(\alpha e)=\alpha \Phi(e)$ for every $\alpha$ in $\mathbf{R}$, where $e=1$ on $[0,1]$. It is also easy to check that (2.2) and (2.3) can be replaced by the single condition:

(2.4) $\Phi(f+g)=\Phi(f)+\Phi(g)$ whenever $g$ is constant on the support of $f$.

If (2.1) is replaced by the stronger condition:

(2.5) $\lim _{\|f-g\|_{\rightarrow 0}} \Phi(f)=\Phi(g)$,

then it is easy to show that (2.2) can be replaced by the weaker condition:

(2.6) $\Phi(f+g)=\Phi(f)+\Phi(g)$ whenever $f$ and $g$ have disjoint closed supports.

In $\$ 3$ we will actually prove the following theorem:

Theorem 2. Let $\Phi: \mathcal{C}_{0}(\mathbf{R}) \rightarrow \mathbf{R}$ be a functional satisfying (2.1) and (2.4). Then $\Phi$ is linear. 
Any functional $\Phi$ on $\mathcal{C}([0,1])$ which satisfies (2.1) and (2.4) obviously induces a functional on $\mathcal{C}_{0}(R)$ with the same properties, so Theorem $2 \mathrm{im}$ plies Theorem 1.

Before proceeding with the proof of Theorem 2, we will justify the remark made earlier, that condition (iii) of $\$ 1$ may be replaced by (iii)'. Let $M$ be any topological space. Let $\Phi$ be a functional on $\mathcal{C}(M)$ that satisfies either (2.1), (2.2) and (2.3) or (2.5), (2.6) and (2.3).

Theorem 3. $\Phi(\alpha f)=\alpha \Phi(f)$ for any $f$ in $\mathcal{C}(M), \alpha$ in $\mathbf{R}$.

Proof. Fix $f$ in $\mathcal{C}(M)$. Let $f(M)$ be contained in the finite interval $[a, b]$. Define $\Gamma$ on $\mathcal{C}([a, b])$ by $\Gamma(b)=\Phi(b \circ f)$. Then $\Gamma$ satisfies the conditions of Theorem 1 and is accordingly linear. In particular $\Gamma(\alpha b)=$ $\alpha \Gamma(h)$ where $h(x)=x$ for all $x$ in $[a, b]$. That is, $\Phi(a f)=\alpha \Phi(f)$.

The corresponding result for operators follows by considering point evaluation again.

\section{Proof of Theorem 2.}

Definition 1. For each $r \geq 0$, let $\phi(r)=\sup |\Phi(f)|$, taken over all $f$ in $\mathcal{C}_{0}(\mathbf{R})$ with $\|f\| \leq r$.

By (2.1), $\lim _{r \rightarrow 0} \phi(r)=0$. In particular $\phi(r)$ is finite for sufficiently small $r$.

Lemma 1. $\phi(r)$ is finite for all $r$.

Proof. Fix $r$. Let $f$ be in $\mathcal{C}_{0}(\mathbf{R})$ with $\|f\| \leq r$.

Let $f^{+}=f \vee 0$ and $f^{-}=f \wedge 0$. Then $\Phi(f)=\Phi\left(f^{+}\right)+\Phi\left(f^{-}\right)$.

Choose $g$ in $\mathcal{C}_{0}(\mathbf{R})$ such that $0 \leq g \leq 1 / 2\left\|f^{+}\right\|$, and such that $g=1 / 2\left\|f^{+}\right\|$ on the support of $f^{+}$. Then $\Phi\left(f^{+}-g\right)=\Phi\left(f^{+}\right)+\Phi(-g)$, so

$$
\left|\Phi\left(f^{+}\right)\right| \leq\left|\Phi\left(f^{+}-g\right)\right|+|\Phi(-g)| \leq 2 \phi(r / 2) \text {. }
$$

Similarly $\left|\Phi\left(f^{-}\right)\right| \leq 2 \phi(r / 2)$. Thus $|\Phi(f)| \leq 4 \phi(r / 2)$. Hence $\phi(r) \leq$ $4 \phi(r / 2)$. Hence $\phi(r) \leq 4^{n} \phi\left(r / 2^{n}\right)$. For large $n, \phi\left(r / 2^{n}\right)$ is already known to be finite, so Lemma 1 is proved.

Lemma 2. Let $f_{n}$ be a uniformly bounded sequence in $\mathcal{C}_{0}(\mathbf{R})$. Each of the following statements implies that $\Phi\left(f_{n}\right) \rightarrow 0$ as $n \rightarrow \infty$ :

(3.1) The $f_{n}$ have mutually disjoint supports.

(3.2) For any compact set $E$, all but finitely many $f_{n}$ vanish on $E$.

Proof. Suppose $\left\|f_{n}\right\| \leq r$ for all $n$. Let (3.1) hold. Let $A_{n}=\{k \mid k=1$, $\left.\cdots, n, \Phi\left(f_{k}\right) \geq 0\right\}$. Let $B_{n}=\{1, \cdots, n\}-A_{n}$. Then 
$\sum_{k=1}^{n}\left|\Phi\left(f_{k}\right)\right|=\sum_{k \in A_{n}} \Phi\left(f_{k}\right)-\sum_{k \in B_{n}} \Phi\left(f_{k}\right)=\Phi\left(\sum_{k \in A_{n}} f_{k}\right)-\Phi\left(\sum_{k \in B_{n}} f_{k}\right) \leq 2 \phi(r)$.

Hence $\Sigma_{k=1}^{\infty}\left|\Phi\left(f_{k}\right)\right|<\infty$, so $\Phi\left(f_{n}\right) \rightarrow 0$ as $n \rightarrow \infty$.

Now suppose (3.2) holds. Let $g_{k}$ be any subsequence of $f_{n^{*}}$. We can choose a subsequence $h_{i}$ from $g_{k}$ such that the $h_{i}$ have mutually disjoint supports. By what has already been proved, $\Phi\left(h_{i}\right) \rightarrow 0$ as $i \rightarrow \infty$. Since $g_{k}$ was arbitrary, we must have $\Phi\left(f_{n}\right) \rightarrow 0$ as $n \rightarrow \infty$. This completes the proof of Lemma 2.

Lemma 3. Let $f_{n}$ and $g_{n}$ be uniformly bounded sequences in $\mathcal{C}_{0}(\mathbf{R})$ such that for any compact set $E, f_{n}-g_{n}$ vanishes on $E$ for all but finitely many $n$. Then $\Phi\left(f_{n}\right)-\Phi\left(g_{n}\right) \rightarrow 0$ as $n \rightarrow \infty$.

Proof. To prove that a sequence of numbers converges to zero, it is enough to show that every subsequence contains another subsequence that converges to zero. Thus, by choosing subsequences and relabelling, we may assume that $f_{n}=g_{n}$ on $[-n, n]$ for all $n$.

Let $h_{n}=f_{n}$ on $(-\infty, 0], h_{n}=g_{n}$ on $[0, \infty)$. Let $p_{n}=1$ on $(-\infty, n)$, $p_{n}=0$ otherwise. Let $q_{n}=1$ on $(n, \infty), q_{n}=0$ otherwise. Let $u_{n}$ be any bounded sequence in $\mathcal{C}_{0}(\mathbf{R})$ such that $u_{n}=f_{n}(n)$ on the union of the supports of $f_{n}$ and $g_{n}$. Then:

$$
\Phi\left(f_{n}-u_{n}\right)=\Phi\left(f_{n}\right)+\Phi\left(-u_{n}\right), \quad \text { and } \quad \Phi\left(b_{n}-u_{n}\right)=\Phi\left(b_{n}\right)+\Phi\left(-u_{n}\right) .
$$

Also

$$
\Phi\left(f_{n}-u_{n}\right)=\Phi\left(p_{n}\left(f_{n}-u_{n}\right)\right)+\Phi\left(q_{n}\left(f_{n}-u_{n}\right)\right)
$$

and

$$
\Phi\left(b_{n}-u_{n}\right)=\Phi\left(p_{n}\left(b_{n}-u_{n}\right)\right)+\Phi\left(q_{n}\left(b_{n}-u_{n}\right)\right)
$$

But $p_{n}\left(f_{n}-u_{n}\right)=p_{n}\left(b_{n}-u_{n}\right)$. We get

$$
\Phi\left(f_{n}\right)-\Phi\left(b_{n}\right)=\Phi\left(q_{n}\left(f_{n}-u_{n}\right)\right)-\Phi\left(q_{n}\left(b_{n}-u_{n}\right)\right) \text {. }
$$

By Lemma 2, $\Phi\left(f_{n}\right)-\Phi\left(b_{n}\right) \rightarrow 0$ as $n \rightarrow \infty$. In the same way $\Phi\left(b_{n}\right)-$ $\dot{\Phi}\left(g_{n}\right) \rightarrow 0$ as $n \rightarrow \infty$, so Lemma 3 is proved.

Let $a$ and $b$ be extended real numbers, $-\infty \leq a<b \leq \infty$. For any function $f$ in $\mathcal{C}((a, b))$ we can choose a uniformly bounded sequence $f_{n}$ in $\mathcal{C}_{0}(\mathbf{R})$ such that each $f_{n}$ has compact support in $(a, b)$ and such that for any compact set $E$ in $(a, b), f_{n}-f$ vanishes on $E$ for all but finitely many 
n. It follows easily from Lemma 3 that $\Phi\left(f_{n}\right)$ converges to a limit, and that this limit is independent of the choice of the sequence $f_{n}$. Define the functional $\Phi_{(a, b)}$ on $\mathcal{C}((a, b))$ by

$$
\Phi_{(a, b)}(f)=\lim _{n \rightarrow \infty} \Phi\left(f_{n}\right)
$$

For $r \geq 0$ let

$$
\begin{gathered}
\phi_{(a, b)}(r)=\sup \left|\Phi_{(a, b)}(f)\right|, \quad \phi_{(a, b)}^{+}(r)=\sup \Phi_{(a, b)}(f), \\
\phi_{(a, b)}^{-}(r)=\sup \left(-\Phi_{(a, b)}(f)\right),
\end{gathered}
$$

where the suprema are taken over all $f$ in $\mathcal{C}((a, b))$ with $\|f\| \leq r$. Clearly

$$
\phi(a, b) \leq \phi \text {. }
$$

Also, for any $c, a<c<b$, we see easily that

$$
\phi_{(a, c)}^{+}+\phi_{(c, b)}^{+} \leq \phi_{(a, b)}^{+}, \quad \phi_{(a, c)}^{-}+\phi_{(c, b)}^{-} \leq \phi_{(a, b)}^{-} .
$$

As noted earlier, Lemma 3 and the resulting extension of $\Phi$ do not hold in higher dimensions. It appears that the best that can be done is to extend $\Phi$ to all continuous functions defined on the compact closure of an open subset $E$, which are constant on each component of the boundary of E.

Lemma 4. $\Phi_{(a, b)}$ satisfies conditions (2.1) and (2.4).

Proof. (2.1) follows from (3.5). Let $f$ and $g$ be in $\mathcal{C}((a, b))$ with $g$ constant on the support of $f$. We can choose a uniformly bounded sequence $f_{n}$ in $\mathcal{C}_{0}(\mathbf{R})$ such that each $f_{n}$ has compact support contained in $(a, b) \cap$ (support $f$ ), and such that for any compact set $E$ in $(a, b), f_{n}-f$ vanishes on $E$ for all but finitely many $n$. We can choose a uniformly bounded sequence $g_{n}$ in $\mathcal{C}_{0}(\mathbf{R})$ such that each $g_{n}$ has compact support in $(a, b)$, such that for any compact set $E$ in $(a, b), g_{n}-g$ vanishes on $E$ for all but finitely many $n$, and such that $g_{n}=g$ on support $f_{n}$. Then $\Phi\left(f_{n}+g_{n}\right)=\Phi\left(f_{n}\right)+$ $\Phi\left(g_{n}\right)$ by (2.4), $\Phi\left(f_{n}\right) \rightarrow \Phi_{(a, b)}(f), \Phi\left(g_{n}\right) \rightarrow \Phi_{(a, b)}(g)$, and $\Phi\left(f_{n}+g_{n}\right) \rightarrow$ $\Phi_{(a, b)}(f+g)$ by definition. This proves Lemma 4 .

Suppose $-\infty \leq a<c<b \leq \infty$. Define $F: \mathcal{C}((a, b)) \rightarrow \mathbf{R}$ by

$$
F(f)=\Phi_{(a, b)}(f)-\Phi_{(a, c)}\left(\left.f\right|_{(a, c)}\right)-\Phi_{(c, b)}\left(\left.f\right|_{(c, b)}\right)
$$




\section{Lemma 5. $F$ is linear.}

Proof. F clearly satisfies conditions (2.1) and (2.4), by Lemma 4. Let $e$ be the function which is 1 on $(a, b)$. Then $F(f)=F(f-f(c) e)+$ $F(f(c) e)$. Thus it is enough to show that $F(f-f(c) e)=0$, or in other words that $F(f)=0$ whenever $f(c)=0$. If $f(c)=0$, let $f=g+h$, where $g=0$ on $[c, b)$ and $h=0$ on $(a, c] . F(f)=F(g)+F(b)$. Hence it is enough to show that $F(g)=0$ whenever $g=0$ on $[c, b)$. That is, to show $\Phi_{(a, b)}(g)=$ $\Phi_{(a, c)}\left(\left.g\right|_{(a, c)}\right)$. Since $F(g)=F(g \vee 0)+F(g \wedge 0)$ we may assume $g \geq 0$.

Choose a uniformly bounded sequence $g_{n}$ in $\mathcal{C}_{0}(\mathbf{R})$ such that each $g_{n}$ has compact support in $(a, c]$, such that for any compact set $E$ in $(a, b)$, $g_{n}-g$ vanishes on $E$ for all but finitely many $n$, and such that $g_{n} \geq 0$ for all $n$. Let $h_{n}$ be a sequence in $\mathcal{C}_{0}(\mathbf{R})$, such that each $h_{n}$ has compact support in $(a, c),\left\|h_{n}\right\| \leq 1 / n$, and $h_{n}=g_{n} \wedge(1 / n)$ on the union of support $\left(g_{n}-g_{n} \wedge(1 / n)\right)$ and $[a+(1 / n), c-(1 / n)]$. Clearly $\Phi_{(a, b)}(g)=$ $\lim _{n \rightarrow \infty} \Phi\left(g_{n}\right)$, by definition.

Since $g_{n}-g_{n} \wedge(1 / n)+h_{n}=g_{n}$ on $[a+(1 / n), c-(1 / n)]$, also

$$
\Phi_{(a, c)}\left(\left.g\right|_{(a, c)}\right)=\lim _{n \rightarrow \infty} \Phi\left(g_{n}-g_{n} \wedge(1 / n)+b_{n}\right),
$$

by definition. But $h_{n}=g_{n} \wedge(1 / n)=1 / n$ on support $\left(g_{n}-g_{n} \wedge(1 / n)\right)$. Thus

$$
\Phi\left(g_{n}-g_{n} \wedge(1 / n)+b_{n}\right)=\Phi\left(g_{n}-g_{n} \wedge(1 / n)\right)+\Phi\left(b_{n}\right)
$$

and

$$
\Phi\left(g_{n}\right)=\Phi\left(g_{n}-g_{n} \wedge(1 / n)\right)+\Phi\left(g_{n} \wedge(1 / n)\right)
$$

Since $\Phi\left(h_{n}\right) \rightarrow 0$ and $\Phi\left(g_{n} \wedge(1 / n)\right) \rightarrow 0$ as $n \rightarrow \infty$ by (2.1), we have $\Phi_{(a, b)}(g)=\Phi_{(a, c)}\left(\left.g\right|_{(a, c)}\right)$, and Lemma 5 is proved.

Lemma 6. Let $f$ and $g$ be in $\mathcal{C}((a, b))$ with $|f(x)-f(y)| \leq \epsilon$ and $|g(x)-g(y)| \leq \epsilon$ for all $x$ and $y$ in $(a, b)$. Then

$$
\left|\Phi_{(a, b)}(f+g)-\Phi_{(a, b)}(f)-\Phi_{(a, b)}(g)\right| \leq 3 \phi_{(a, b)}(2 \epsilon) .
$$

Proof. Choose $c$ in $(a, b)$. Let $e=1$ on $(a, b)$. Then

$$
\begin{aligned}
\Phi_{(a, b)}(f+g)-\Phi_{(a, b)}(f)-\Phi_{(a, b)}(g) & =\Phi_{(a, b)}(f+g-f(c) e-g(c) e) \\
& -\Phi_{(a, b)}(f-f(c) e)-\Phi_{(a, b)}(g-g(c) e) .
\end{aligned}
$$

Lemma 6 follows from the definition of $\phi_{(a, b)}$.

Lemma 7. $\Phi$ is additive. 
Proof. Let $f$ and $g$ be in $\mathcal{C}_{0}(\mathbf{R})$. Let $\epsilon>0$ be given. Choose $a_{0}, \cdots$, $a_{n}$ such that $-\infty=a_{0}<a_{1}<\cdots<a_{n}=\infty$, and such that $|f(x)-f(y)| \leq \epsilon$, $|g(x)-g(y)| \leq \epsilon$, for all $x$ and $y$ in $\left(a_{i}, a_{i+1}\right), i=0, \ldots, n-1$.

Define $G$ on $\mathcal{C}_{0}(\mathbf{R})$ by

$$
G(b)=\sum_{i=0}^{n-1} \Phi\left(a_{i}, a_{i+1}\right)\left(\left.b\right|_{\left(a_{i}, a_{i+1}\right)}\right), \text { for every } b \text { in } \mathcal{C}_{0}(\mathbf{R})
$$

It follows from Lemma 5 that $\Phi-G$ is linear. Hence

$$
\Phi(f+g)-\Phi(f)-\Phi(g)=G(f+g)-G(f)-G(g)
$$

By Lemma 6,

$$
\begin{aligned}
|\Phi(f+g)-\Phi(f)-\Phi(g)| & \leq \sum_{i=0}^{n-1} 3 \phi_{\left(a_{i}, a_{i+1}\right)}(2 \epsilon) \\
& \leq \sum_{i=0}^{n-1} 3\left[\phi_{\left(a_{i}, a_{i+1}\right)}^{+}(2 \epsilon)+\phi_{\left(a_{i}, a_{i+1}\right)}^{-}(2 \epsilon)\right] \\
& \leq 3\left[\phi_{(-\infty, \infty)}^{+}(2 \epsilon)+\phi_{(-\infty, \infty)}^{-}(2 \epsilon)\right] \text { by }(3.6) \\
& \leq 6 \phi_{(-\infty, \infty)}(2 \epsilon) \leq 6 \phi(2 \epsilon) \text { by }(3.5) .
\end{aligned}
$$

This proves Lemma 7.

The proof of Theorem 2 is now immediate, since Lemma 7 enables us to deduce (2.5) (continuity everywhere) from (2.1) (continuity at the origin). Continuity plus additivity then gives linearity, in the usual way.

4. Weak convergence of measures. As an application of Theorem 3, we prove

Theorem 4. Let $M$ be a topological space. Let $\mu_{n}$ be a sequence of signed Borel measures on $M$, such that:

(4.1) $\left\|\mu_{n}\right\|$ is uniformly bounded,

(4.2) $\lim \sup _{n \rightarrow \infty} \int(f+g) d \mu_{n}=\lim \sup _{n \rightarrow \infty} \int f d \mu_{n}+\lim \sup _{n \rightarrow \infty} \int g d \mu_{n}$, for all $f$ and $g$ in $\mathcal{C}(M)$ with disjoint closed support,

(4.3) $\lim _{n \rightarrow \infty} \mu_{n}(M)$ exists. Then $\lim _{n \rightarrow \infty} \int f d \mu_{n}$ exists for all $f$ in $\mathcal{C}(M$ :

Proof. Define the functional $\Phi$ on $\mathcal{C}(M)$ by $\Phi(f)=\lim \sup \int f d \mu_{n}$. It is easy to see that $\Phi$ satisfies (2.5), (2.6) and (2.3). Hence $\Phi(f)=-\Phi(-f)$ for every $f$, by Theorem 3. But $-\Phi(-f)=\lim \inf \int f d \mu_{n}$, so Theorem 4 is proved. 


\section{REFERENCES}

1. R. V. Chacon and N. A. Friedman, Additive functionals, Arch. Rational Mech. Anal. 18 (1965), 230-240. MR 30 \#2329.

2. N. A. Friedman and M. Katz, A representation theorem for additive functionals, Arch. Rational Mech. Anal. 21 (1965), 49-57. MR 33 \#245.

3. - Additive functionals on $L_{p}$ spaces, Canad. J. Math. 18 (1966), 1264-1271. MR 34 \#6510.

4. - On additive functionals, Proc. Amer. Math. Soc. 21 (1969), 557561. MR 39 \#4653.

5. N. A. Friedman and A. E. Tong, On additive operators, Canad. J. Math. 23 (1971), 468-480. MR $44 \# 785$.

DEPARTMENT OF MATHEMATICS, UNIVERSITY OF MINNESOTA, MINNEAPOLIS, MINNESOT A 55455 